\title{
A COMPARATIVE STUDY OF LOCUS AND OTHER MANY CORE ARCHITECTURES BASED ON IOT
}

\author{
Jasleen Kaur $^{1}$ and Anil Kumar ${ }^{2}$
}

\begin{abstract}
This paper represents LOCUS - a low-power, customizable, many-core processor for next-generation wearable devices. LOCUS combines customizable processor cores with a customizable network on a message-passing architecture. The architecture of LOCUS is such that it can be implemented in any kind of application ranging from medical field to the commercial ones. The Many-core architectures evolve when everyday ordinary objects have interconnected microchips inside them. It also discuss the comparison of various techniques related to the processor architecture based on no of parameters which has shown that the performance and speedup of the processor architecture is high whereas the energy consumption is low.

Keywords- Many core architecture, LOCUS, customizable cores, energy efficient processors, low power, customizable network
\end{abstract}

\section{INTRODUCTION}

The whole of interrelated computing devices, mechanized as well as digital devices, objects, flora and fauna or even individuals who are given with distinct identifiers as well as the capacity to exchange data within network without having the need interaction of human-to-computer or human-to-human is known as IoT(Internet of Things). In IoT, a thing may be an individual having a cardiovascular detector implant, a village animal having a biochip detector, a vehicle which is having in-built detectors which notify the driver whenever the pressure of tire is minimal - or any artificial or any natural thing that can be given an IP address and help in exchange of info over the network.

\section{A. Wearable}

It is a category of technological innovation gadgets which can be worn out by anyone and also many times includes monitoring info associated with physical fitness and health. It also comprises gadgets which contains small movement detectors to capture images and synchronize with all the mobile devices. Wearable gadgets like activity trackers make a perfect demonstration of the Internet of Things, being that they are portion of the network involving real objects or "things" inlayed within electronic devices or gadgets, software, sensors as well as connectivity for allowing the connected things to interchange info with anyone without requiring human intervention.

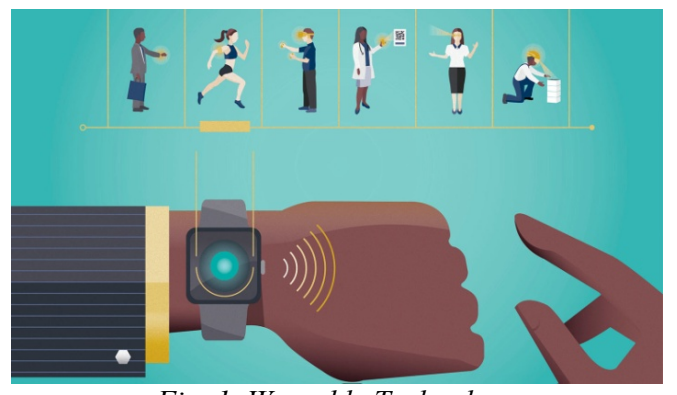

\section{B. Types of Processor Architecture \\ 1) Multi-core Processors}

Fig. 1. Wearable Technology

A single computing component using at least two independent cores(actual processing units) that are used to read as well as execute different program instructions is a multi-core processor. These instructions run by a multi core processor are simple CPU instructions. The multi core processor increases the overall speed of the programs. Cores can be coupled loosely or

\footnotetext{
${ }^{1}$ Department of Computer Engineering \& Technology, Guru Nanak Dev University, Amritsar, India

${ }^{2}$ Department of Computer Engineering \& Technology, Guru Nanak Dev University, Amritsar, India
} 
tightly depending on the needs of the designers. For instance, caches may or not be shared among different caches as well as different methods of communication may be implemented by different cores. Two-dimensional mesh, bus, crossbar as well as ring are among the general network topologies adopted for interconnecting cores. The multi-core systems which are homogeneous in nature consist of identical cores; whereas those which are heterogeneous comprise the cores which are not identical.

\section{2) Many-core Processors}

The particular multi-core processors intended for a very great level of concurrent processing as well as that incorporate numerous independent processor cores are known as ManyCore processors. Embedded computers and high-performance computing relies extensively on ManyCore processors. They are not the same as multi-core processors since they are optimized from the beginning for an increased level of explicit parallelism, and then for greater throughput/performance or reduced energy utilization at the cost of latency along with lower single thread performance. A ManyCore processor using a network on a chip and local memories provides software the ability to explicitly enhance the spatial design of tasks.

\section{RELATED WORK}

F.Conti et al 2015[2] proposed PULP which is parallel accelerator and a ultra-low power in design specially intended for increasing energy-efficiency as well as providing flexible embedded vision. PULP design relies upon clusters involving tightlycoupled OpenRISC ISA cores. C.H.O Chen et al. 2013[3] has suggested SMART (Single-cycle Multi-hop Asynchronous Repeated Traversal) NoC, a NoC which at run time reconfigures plus tailors a normal mesh topology intended for SoC applications. A new low-swing clockless link circuit included in the router crossbars is the heart of SMART NoC. L. Chen et al. 2013 [4] has proposed an architecture known as Just-in-Time Customizable or JiTC processor which resolves the contradictory requirements of efficiency as well as flexibility, that need to be resolved in extensible processors. The most important advancement in JiTC is a multi-stage accelerator, which is also known as SFU (Specialized Functional Unit), which is integrated firmly within the processor-pipeline. T. Krishna et al. 2013 [5] has worked on scalable on-chip topologies like meshes which considerably add many hops in each traversal of network as the number of on-chip cores increase. An on-chip network which is called SMART(Single-cycle Multi-hop Asynchronous Repeated Traversal) is proposed that presents a single-cycle data-path starting from the source till the destination. A.Y. Dogan et al. 2012 [6] has proposed that the near-threshold ultra-low-power multi-core design which displays low-power cores which is competent of carrying out biomedical applications, having numerous instruction as well as data memories which are coupled tightly with the help of flexible crossbar interconnects. L. Gwennap et al. 2011[7] has offered Floating-Point Accelerator for Mobile Processors. The frequent measure of power efficiency is MIPS per watt and most of the processor vendors emphasize only on this. The Epiphany multicore architecture is offered as an intellectual-property (IP) core by Adapteva. The fundamental 16-core design is rated at 19Gflops by the company . S.V. Tota et al. 2010[9] has proposed MEDEA which is an NoC-based framework [9]. Medea is a hybrid message-passing/shared-memory approach [9]. S. Li et al. 2009 [10] introduced McPAT which is an integrated area, power as well as timing modeling framework that can be used for extensive design space research with regard to manycore and also multicore model designs. McPAT has an extensible XML interface for facilitating its use with many performance simulators. N. Agarwal et al. 2009 [11] have proposed GARNET which is a precise on-chip network model. The authors have developed GARNET which is a comprehensive cycleaccurate interconnection network design. In the implementation of GARNET, the GEMS full-system simulation framework is used [11]. Microarchitectural details, for example routing logic, flit-level input buffers, the crossbar switch and allocators are designed. P.Yu and T.Mitra et al. 2004 [16] have advised the adding up of custom instructions which are application-specific to the core instruction set architecture is allowed in extensible processors. An effective algorithm to get specific enumeration of the feasible candidate instructions is suggested.

\section{TABLE OF COMPARISON}

\begin{tabular}{|c|c|c|c|c|c|c|c|}
\hline Author Name & Year & Paper Title & Technique Used & \multicolumn{4}{|c|}{ Parameters } \\
\hline Tan Cheng et al & 2016 & $\begin{array}{l}\text { LOCUS: low-power } \\
\text { customizable many- } \\
\text { core architecture for } \\
\text { wearables .[1] }\end{array}$ & $\begin{array}{l}\text { Customizable Processor } \\
\text { Cores implemented on } \\
\text { customizable network \& } \\
\text { message passing } \\
\text { architecture [1] }\end{array}$ & $\checkmark$ & $\checkmark$ & $\checkmark$ & $\checkmark$ \\
\hline F.Conti et al & 2015 & $\begin{array}{lr}\text { PULP: A ultra-low } \\
\text { power parallel } \\
\text { accelerator } r \text { for } \\
\text { energy-efficient and } \\
\text { flexible embedded } \\
\text { vision[2] }\end{array}$ & $\begin{array}{l}\text { Implemented ring } \\
\text { tightly-coupled clusters } \\
\text { of OpenRISC ISA cores } \\
{[2]}\end{array}$ & $\checkmark$ & $x$ & $\checkmark$ & $\checkmark$ \\
\hline C.H.O Chen et al. & 2013 & $\begin{array}{l}\text { SMART: a single- } \\
\text { cycle reconfigurable }\end{array}$ & $\begin{array}{l}\begin{array}{l}\text { Low-swing } \\
\text { repeated link }\end{array} \begin{array}{r}\text { clockless } \\
\text { circuit }\end{array} \\
\end{array}$ & $\checkmark$ & $x$ & $\checkmark$ & $\checkmark$ \\
\hline
\end{tabular}




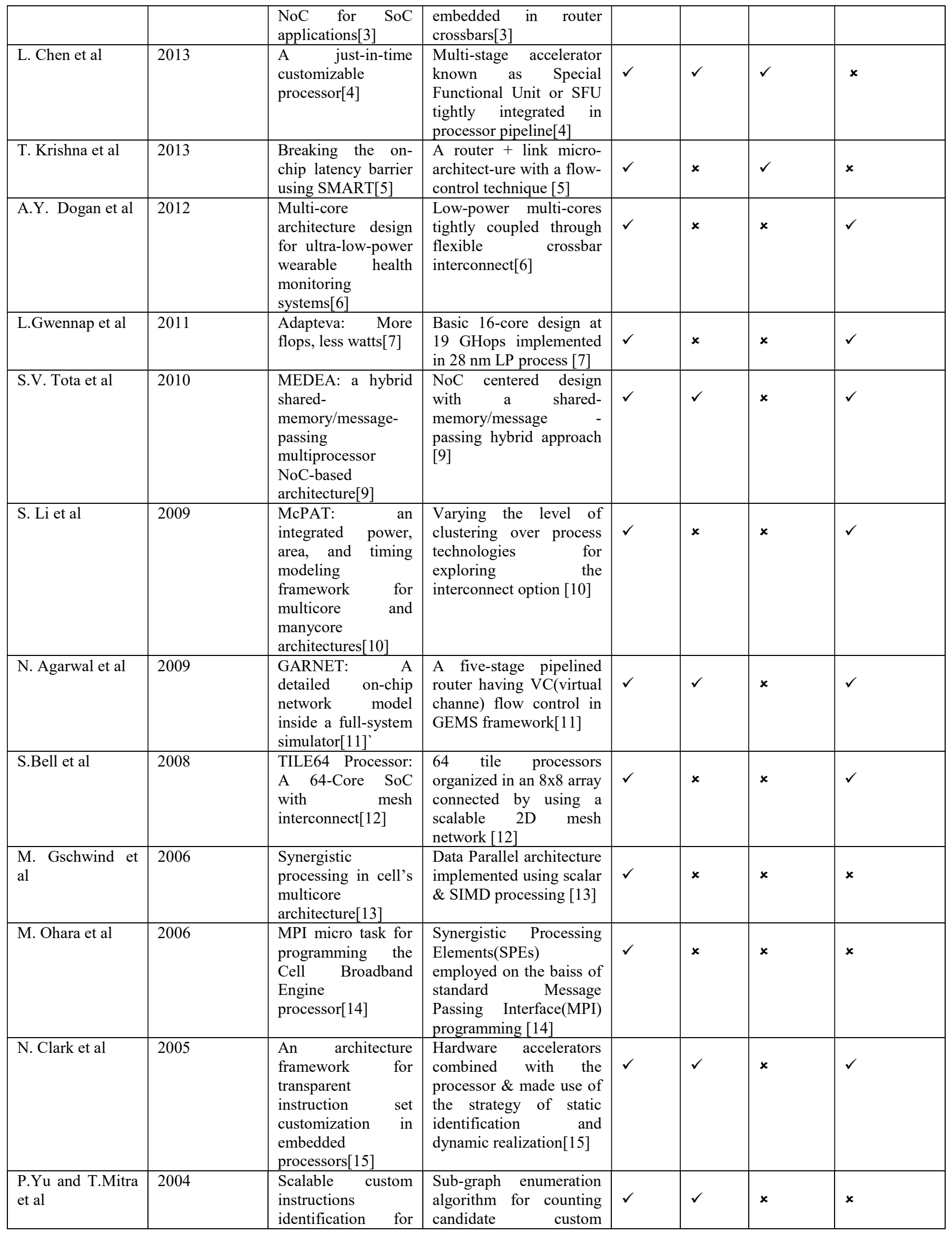




\begin{tabular}{|l|l|l|l|l|l|l|}
\hline & $\begin{array}{l}\text { instruction-set } \\
\text { extensible } \\
\text { processors[16] }\end{array}$ & instructions [16] & & & \\
\hline
\end{tabular}

\section{CONCLUSION}

The requirements and demands of applications, such as real-time response, are pushing the wearable devices to leverage more power-efficient processors inside the SoC (System-on-chip). So LOCUS combines customizable processor cores with a customizable network on a message-passing architecture. So in this paper, it represnts the comparisons of various processor architecture by taking various parameters in which all parameters perform well but in case of core failure in computing of critical data, the system will lose its accuracy. So the effect of failures in many core computing has been ignored by the existing literature. So in near future we will propose HALOCUS- Highly Available LOCUS which will make use of fuzzy logic in case of core failure.

\section{REFERENCES}

[1] Bowman, Tan, Cheng, Aditi Kulkarni, Vanchinathan Venkataramani, Manupa Karunaratne, Tulika Mitra, and Li-Shiuan Peh.: LOCUS: low-power customizable many-core architecture for wearables. In Proceedings of the International Conference on Compilers, Architectures and Synthesis for Embedded Systems, pp. 11. ACM, (2016)

[2] Conti, Francesco, Davide Rossi, Antonio Pullini, Igor Loi, and Luca Benini.: PULP: A Ultra-Low Power Parallel Accelerator for Energy-Efficient and Flexible Embedded Vision. Journal of Signal Processing Systems,pp.1-16 (2015)

[3] Chen, Chia-Hsin Owen, Sunghyun Park, Tushar Krishna, Suvinay Subramanian, Anantha P. Chandrakasan, and Li-Shiuan Peh.:SMART: a single-cycle reconfigurable NoC for SoC applications. In Proceedings of the Conference on Design, Automation and Test in Europe, pp. 338-343. EDA Consortium(2013)

[4] Chen, Liang, Joseph Tarango, Tulika Mitra, and Philip Brisk.: A just-in-time customizable processor.In 2013 IEEE/ACM International Conference on Computer-Aided Design (ICCAD), pp. 524-531. IEEE(2013)

[5] Krishna, Tushar, Chia-Hsin Owen Chen, Woo Cheol Kwon, and Li-Shiuan Peh.: Breaking the on-chip latency barrier using SMART. In High Performance Computer Architecture (HPCA2013), 2013 IEEE 19th International Symposium on, pp. 378-389. IEEE(2013)

[6] Dogan, Ahmed Yasir, Jeremy Constantin, Martino Ruggiero, Andreas Burg, and David Atienza.: Multi-core architecture design for ultra-low-power wearable health monitoring systems. In Proceedings of the Conference on Design, Automation and Test in Europe, pp. 988-993. EDA Consortium(2012)

[7] Gwennap, Linley.: Adapteva: More flops, less watts. Microprocessor Report 6, no. 13: 11-02(2011)

[8] Ma, Kai, Xue Li, Ming Chen, and Xiaorui Wang.: Scalable power control for many-core architectures running multi-threaded applications. In ACM SIGARCH Computer Architecture News, vol. 39, no. 3, pp. 449-460. ACM(2011)

[9] Tota, Sergio V., Mario R. Casu, Massimo Ruo Roch, Luca Rostagno, and Maurizio Zamboni.: MEDEA: a hybrid shared-memory/message-passing multiprocessor NoC-based architecture. In 2010 Design, Automation \& Test in Europe Conference \& Exhibition (DATE 2010), pp. 45-50. IEEE(2010)

[10] Li, Sheng, Jung Ho Ahn, Richard D. Strong, Jay B. Brockman, Dean M. Tullsen, and Norman P. Jouppi.: McPAT: an integrated power, area, and timing modeling framework for multicore and manycore architectures. In Proceedings of the 42nd Annual IEEE/ACM International Symposium on Microarchitecture, pp. 469-480. ACM(2009)

[11] Agarwal, Niket, Tushar Krishna, Li-Shiuan Peh, and Niraj K. Jha.: GARNET: A detailed on-chip network model inside a full-system simulator. In Performance Analysis of Systems and Software, 2009. ISPASS 2009. IEEE International Symposium on, pp. 33-42. IEEE(2009)

[12] Bell, Shane, Bruce Edwards, John Amann, Rich Conlin, Kevin Joyce, Vince Leung, John MacKay, J., Reif, M., Bao, L., Brown, J. and Mattina, M.: Tile64-processor: A 64-core soc with mesh interconnect. In 2008 IEEE International Solid-State Circuits Conference-Digest of Technical Papers, pp. 88598. $\operatorname{IEEE}(2008)$

[13] Gschwind, Michael, H. Peter Hofstee, Brian Flachs, Martin Hopkins, Yukio Watanabe, and Takeshi Yamazaki.: Synergistic processing in Cell's multicore architecture. IEEE micro 26, no. 2,pp. 10-24(2006).

[14] Ohara, Moriyoshi, Hiroshi Inoue, Yukihiko Sohda, Hideaki Komatsu, and Toshio Nakatani. "MPI microtask for programming the Cell Broadband Engine $^{\mathrm{TM}}$ processor." IBM Systems Journal 45, no. 1,pp. 85-102 (2006)

[15] Clark, Nathan, Jason Blome, Michael Chu, Scott Mahlke, Stuart Biles, and Krisztian Flautner.: An architecture framework for transparent instruction set customization in embedded processors. In ACM SIGARCH Computer Architecture News, vol. 33, no. 2, pp. 272-283. IEEE Computer Society(2005)

[16] Yu, Pan, and Tulika Mitra.: Scalable custom instructions identification for instruction-set extensible processors. In Proceedings of the 2004 international conference on Compilers, architecture, and synthesis for embedded systems, pp. 69-78. ACM(2004)

[17] Yu, Pan, and Tulika Mitra.: Characterizing embedded applications for instruction-set extensible processors. In Proceedings of the 41st annual Design Automation Conference, pp. 723-728. ACM(2004)

[18] Clark, Nathan, Manjunath Kudlur, Hyunchul Park, Scott Mahlke, and Krisztian Flautner.: Application-specific processing on a general-purpose core via transparent instruction set customization. In Microarchitecture, 2004. MICRO-37 2004. 37th International Symposium on, pp. 30-40. IEEE(2004)

[19] Duller, Andrew, Gajinder Panesar, and Daniel Towner.: Parallel Processing-the picoChip way. Communicating Processing Architectures 2003,pp. 125138(2003).

[20] Taylor, Michael Bedford, Jason Kim, Jason Miller, David Wentzlaff, Fae Ghodrat, Ben Greenwald, Henry Hoffman, H., Johnson, P., Lee, J.W., Lee, W. and Ma, A.1.: The Raw microprocessor: A computational fabric for software circuits and general-purpose programs. IEEE micro 22, no. 2,pp. 2535(2002) 